\title{
Mineração de Dados aplicada à Saúde Mental de Estudantes Universitários: Uma Revisão Sistemática
}

\author{
Mariana de Lira Farias $^{1}$, Renê Pereira de Gusmão ${ }^{1}$, Cleonides Silva Dias Gusmão ${ }^{1}$ \\ ${ }^{1}$ Departamento de Computação- Universidade Federal de Sergipe (UFS) \\ São Cristóvão - SE - Brazil \\ \{marianalf,rene\}@dcomp.ufs.br, cleonides_silva@hotmail.com
}

\begin{abstract}
This work presents a systematic review intending to evaluate the current state of the art regarding the application of data mining techniques for investigation of mental disorders in university students. Taken into account the use of search strings, 187 papers were found. Besides, as a result of the abstracts reading as well as the application of the inclusion and exclusion criteria, 53 works were selected for further complete analysis. Finally, 25 papers have been accepted for data extraction. Accordingly, herein it was possible to identify the most commonly used algorithm and techniques, mental disorders and related variables, key devices and sample nationalities. Among the most studied disorders, anxiety and depression stand out. These were studied, mainly, by the SVM, neuro fuzzy and KNN techniques. In relation to Brazilian university students, it is possible to highlight the scarcity of studies in relation to mental health, using data mining techniques.
\end{abstract}

Resumo. Este trabalho apresenta uma revisão sistemática de literatura para verificar o estado atual da aplicação de técnicas de mineração de dados para investigação de transtornos mentais em estudantes universitários. Com a utilização de strings de busca, foram encontrados 187 artigos. Após a leitura dos resumos e aplicação dos critérios de inclusão e exclusão, 53 trabalhos foram selecionados para a leitura completa. Ao final, 25 artigos foram aceitos para a extração dos dados. Assim, foi possível verificar quais os algoritmos e técnicas mais utilizados, os transtornos mais frequentes, os principais instrumentos, as variáveis associadas aos transtornos e nacionalidade das amostras. Dentre os transtornos mais estudados, destaca-se a ansiedade e depressão. Para estes, as técnicas mais utilizadas foram SVM, Inferência Neuro Fuzzy e KNN. Em relação a estudantes universitários brasileiros, pode-se perceber uma escassez de estudos em relação a saúde mental com a utilização de técnicas de mineração de dados.

\section{Introdução}

Não existe saúde sem saúde mental. A afirmação comprova que a saúde mental é o cerne do bem estar dos indivíduos e à medida que cresce esse discernimento, torna-se evidente a necessidade de dar enfoque a esse tema [Prince et al. 2007]. Um relatório divulgado em 2017 , constatou que cerca de $10,7 \%$ da população mundial apresenta algum transtorno mental, a proporção é de uma em cada dez pessoas no mundo [Ritchie and Roser 2019].

O cenário também é refletido dentro do ambiente acadêmico. Segundo [Yuan 2014], as universidades estão sofrendo com aumento no número de indivíduos com 
transtornos mentais, pois o ingresso à universidade traz consigo uma exposição a diversas situações, tais como: pressão da família e das matérias curriculares, carga horária e outros fatores, que podem levar a um desequilíbrio na saúde mental dos estudantes.

Dos transtornos mentais, pode-se destacar a ansiedade e a depressão. Os transtornos de ansiedade são caracterizados por medo excessivo e outras perturbações comportamentais correlacionados [Association et al. 2014], enquanto a depressão é caracterizada por tristeza persistente, acompanhada por perturbações somáticas e cognitivas que ocasionam desinteresse e incapacidade de realizar atividades diárias [BRASIL 2016] [Association et al. 2014]. Estima-se que em 2017, cerca de 284 milhões de pessoas experimentaram um transtorno de ansiedade, enquanto, 264 milhões experimentaram a depressão[Ritchie and Roser 2019].

Esse contexto enfatiza a importância de pesquisas sobre os diversos fenômenos ligados a saúde mental, a fim de proporcionar um melhor entendimento sobre o tema e o desenvolvimento de novas técnicas de intervenções e diagnósticos [OMS 2002]. [de Rezende et al. 2008] afirmam que a saúde mental do estudante universitário é foco de pesquisa de muitos especialistas. Dentre os estudos realizados, pode-se citar a utilização de técnicas de mineração de dados e aprendizagem de máquina.

A mineração de dados é o processo de extrair os relacionamentos em grandes conjuntos de dados através da descoberta de fatos por meio de observação e experimentação [Srividya et al. 2018]. Essas técnicas, sobretudo a aprendizagem de máquina, podem contribuir de forma significativa em pesquisas de saúde mental e possibilitar a abertura de áreas completamente novas. Atualmente, pode-se observar diversas aplicações de aprendizagem de máquina e mineração de dados em pesquisas de saúde mental, por exemplo, a utilização de aprendizagem de máquina para analisar o histórico de pacientes, diagnóstico e classificação de distúrbios psiquiátricos, a incidência de um determinado distúrbio em grupo de pessoas [Alonso et al. 2018].

Levando em conta tais aspectos, o presente estudo tem por objetivo apresentar uma revisão bibliográfica da literatura acerca do estado da arte da aplicação das técnicas de mineração de dados para investigação de transtornos mentais em estudantes universitários. Para isso, o artigo está dividido nas seguintes seções: Trabalhos Relacionados, Processo Sistemático, Condução da Revisão Sistemática, Resultados e Discussões, e, por fim, as Conclusões da pesquisa.

\section{Trabalhos Relacionados}

Nesta seção, serão descritos alguns trabalhos relacionados . Em [Cui Yuan 2014] foi realizada a aplicação de técnicas de mineração em uma amostra de 500 estudantes universitários com o objetivo de extrair informações sobre saúde mental para avaliar a vida acadêmica, perspectiva e valores de vida e os relacionamentos interpessoais. Já em [Ahujaa and Bangab 2019], os pesquisadores analisaram o estresse nos estudantes universitários em diferentes momentos de sua vida, a fim de buscar associação com o tempo gasto na internet e os efeitos na mente do aluno.

Em [Pintelas et al. 2018] foi realizada uma revisão de literatura para mapear pesquisas voltadas a previsão de tipos específicos de transtornos de ansiedade, usando técnicas de aprendizado de máquina. [Alonso et al. 2018] também realizou uma re- 
visão sistemática com esse mesmo objetivo, tendo seu foco direcionado a previsão da Demência, Alzheimer, Esquizofrenia e Depressão.

\section{Processo Sistemático}

Nesta seção, serão descritas as etapas do desenvolvimento da Revisão Sistemática: Objetivo e Questões de Pesquisa, Definição das Strings de Busca, Base de Dados, Critérios de Inclusão e Exclusão, Extração de Dados.

\subsection{Objetivo e Questões de Pesquisa}

O objetivo dessa pesquisa é identificar a aplicação das técnicas de mineração de dados para investigação de transtornos mentais em estudantes universitários. Sendo assim, tais questões foram levantadas:

1. Quais os transtornos mentais mais comuns em estudantes universitários?

2. Quais as técnicas de mineração de dados mais utilizadas para identificar transtornos mentais em estudantes universitários?

3. Quais os instrumentos mais utilizados para identificar os transtornos mentais?

4. Quais as variáveis associadas a cada transtorno mental?

5. Quais a nacionalidade dos participantes das amostras?

\subsection{Definição das Strings de Busca}

Antes da construção das Strings de busca, primeiro foi realizada a seleção das palavraschaves utilizadas na pesquisa. As palavras, em um primeiro momento, estavam traduzidas tanto para o português quanto para o inglês e podem ser vizualizadas na tabela 1 .

\begin{tabular}{|l|c|}
\hline \multicolumn{1}{|c|}{ Palavra-Chave } & Sinônimos em Inglês \\
\hline Saúde Mental & Mental Health \\
\hline Ansiedade & Anxiety \\
\hline Depressão & Depression \\
\hline Transtrorno Mental Comum & Common Mental Disorder \\
\hline Mineração de Dados & Data Mining \\
\hline Aprendizagem de Máquina & Machine Learning \\
\hline Cência de Dados & Data Science \\
\hline Técnicas & Techniques \\
\hline Algoritmos & Algorithms \\
\hline
\end{tabular}

Tabela 1. Palavras-Chaves utilizadas nas strings de busca

As strings de busca foram desenvolvidas através da combinação das palavraschaves acima descritas, como pode ser observado na tabela 2.

\subsection{Base de Dados}

No primeiro momento, as bases escolhidas foram Scielo e IEEE Xplore Digital Library, porém devido a insuficiência de artigos relevantes na primeira base, se fez necessário incluir Science Direct. 


\begin{tabular}{|c|}
\hline Strings de Busca \\
\hline student AND ( mental health) AND ( machine learning OR algorithm ) \\
\hline student AND ( mental health OR anxiety OR depression OR common \\
mental disorder ) AND ( algorithms ) \\
\hline student AND ( mental health OR anxiety OR depression OR common \\
mental disorder ) AND ( data mining ) AND ( techniques OR algorithms \\
\hline student AND ( anxiety OR depression ) AND ( data mining OR machine \\
learning OR data science ) AND ( techniques OR algorithms ) \\
\hline student AND ( common mental disorder) AND (data mining OR \\
machine learning OR data science) AND ( techniques OR algorithms ) \\
\hline student AND ( mental health OR anxiety OR depression OR common \\
mental disorder ) AND ( machine AND learning ) \\
\hline student AND ( mental health OR anxiety) AND ( data mining OR \\
machine learning OR data science) AND ( techniques OR algorithms ) \\
\hline student AND ( mental health OR depression ) AND ( data mining OR \\
machine learning OR data science) AND ( techniques OR algorithms ) \\
\hline student AND ( mental health OR anxiety OR depression \\
OR common mental disorder ) AND ( data mining OR machine learning \\
OR data science ) AND ( techniques OR algorithms) \\
\hline student AND (mental health OR anxiety OR depression OR common \\
mental disorder) AND (machine learning) AND (techniques OR \\
algorithms) \\
\hline student AND (mental health) AND (data mining OR machine learning \\
OR data science) AND (techniques OR algorithms) \\
\hline
\end{tabular}

Tabela 2. Strings utilizadas para realizar as buscas nas bases eletrônicas

\subsection{Critérios de Inclusão e Exclusão}

Os critérios de Exclusão e Inclusão podem ser vistos abaixo. Para que um artigo fosse selecionado, o mesmo deveria apresentar todos os critérios de inclusão.

Os critérios de Exclusão foram:

1. Trabalhos que não utilizaram técnicas de mineração de dados ou estatística.

2. Trabalhos que não investigaram a saúde mental de estudantes universitários.

Os Critérios de Inclusão foram:

1. Trabalhos que investigaram a saúde mental de estudantes universitários.

2. Trabalhos publicados entre os anos de 2009-2019.

3. Trabalhos que utilizaram técnicas de mineração de dados ou estatísticas.

\subsection{Procedimento de Seleção de Estudos}

Os procedimentos de seleção de estudos foram divididos em 3 etapas, conforme descritas abaixo:

- Etapa 1: Correspondeu na coleta de estudos através da submissão das strings de buscas nos mecanismos de busca das base selecionadas. É importante destacar que se utilizou a ferramenta de busca avançada e as strings foram adaptadas as particularidades de cada base de dados. 
- Etapa 2: Compreendeu a leitura dos resumos dos artigos e aplicação dos critérios de Inclusão e Exclusão. Assim, os estudos que apresentavam todos os critérios de inclusão foram pré-selecionados para a última fase.

- Etapa 3: Consistiu na retirada de estudos duplicados, leitura integral dos artigos, extração dos dados e resolução das questões de pesquisa.

\subsection{Extração de dados}

Para extração dos dados dos artigos selecionados, as variváveis analisadas foram: Técnicas e Algoritmos, Transtorno Mental, Instrumento, Nacionalidade da Amostra e Variáveis Associadas.

\section{Condução da Revisão Sistemática}

Nesta seção são apresentados os resultados obtidos em cada etapa do procedimento de seleção de estudos. Observe a tabela 3:

\begin{tabular}{|c|c|c|c|}
\hline Base & Etapa 1 & Etapa 2 & Etapa 3 \\
\hline IEEE & 98 & 43 & 14 \\
\hline Science Direct & 89 & 10 & 10 \\
\hline Scielo & 0 & 0 & 0 \\
\hline Total & 187 & 53 & 25 \\
\hline
\end{tabular}

Tabela 3. Resultados das buscas

1

\section{Resultados e Discussões}

Nesta seção estão os resultados e discussões dos dados encontrados. Um ponto a ser destacadado é que, apesar da saúde mental ser foco de pesquisa de muitos espcialistas [de Rezende et al. 2008], há escassez de estudos que trabalhem esse tema aliado a aplicação de técnicas de mineração de dados. A seguir estão as respostas das questões de pesquisa.

\subsection{Quais os transtornos mentais mais comuns em estudantes universitários?}

Em relação aos transtornos encontrados, cerca de 58\% dos trabalhos encontrados tem a ansiedade e a depressão como foco de estudo. Destes, $16 \%$ dos artigos têm depressão como transtorno mental estudado, $16 \%$ tem ansiedade e $25 \%$ dos trabalhos focam em ambos.[da Victoria et al. 2015] afirmam que ansiedade e depressão são os transtornos mais comuns porque estudantes estão expostos as diferentes pressões (relatadas anteriormente) dentro do ambiente universitário que contribuem para o surgimento dos mesmos.

Outro fato identificado, é a porcentagem de estudos que têm como foco o estresse (cerca de 16\%). Apesar deste não ser definido como um transtorno mental, é um fenômeno de interesse nas pesquisas de saúde mental em estudantes universitários, pois alteração nos níveis de estresse podem acarretar em transtornos como depressão, ataque cardíaco e derrame [Ahujaa and Bangab 2019]. Atualmente, o estresse mental é uma

\footnotetext{
${ }^{1}$ IEEE: <http://ieeexplore.ieee.org>, Scielo: <https://scielo.org/ e Science Direct: <https://www.sciencedirect.com/>
} 
questão de grande importância entre os discentes devido a grande quantidade de jovens detectados com esse mal [Ahujaa and Bangab 2019]. Na figura 1 podem ser observadas detalhadamente as porcentagens de transtornos identificados nos artigos analisados.

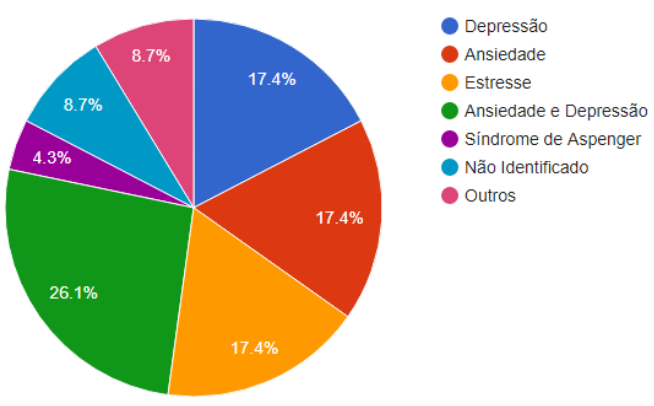

Figura 1. Porcentagem dos Transtornos Encontrados ou Variáveis Associadas

\subsection{Quais as técnicas de mineração de dados mais utilizadas para identificar transtornos mentais em estudantes universitários?}

De forma geral, das técnicas e algoritmos utilizados, pode-se perceber que os algoritmos com abordagem de aprendizado de máquina supervisionado são os mais utilizados. Nessa abordagem, é realizada a previsão de uma variável a partir de uma lista de variáveis independentes [Han and Kamber 2005].

Nos artigos analisados, os algoritmos mais utilizados são: Máquina de Vetores de Suporte (SVM) (cerca de 46\% dos artigos analisados) e Florestas Aleatórias (Random Forest). Máquina de vetores de suporte (SVM) é um algoritmo de aprendizagem supervisionada que analisa e reconhece padrões de um conjunto de dados e normalmente é utilizado para classificação ou regressão [Pintelas et al. 2018]. Já o algoritmo de Florestas Aleatórias, também classicado como técnica de aprendizagem supervisionada, realiza a criação de árvores de decisões - pode ser entendido como um mapa com resultados de decisões tomadas em sequência - e combinações entre as mesmas, a fim de obter a predição com maior acurácia e establidade [Alonso et al. 2018].

Outros algoritmos que merecem destaque são: Algoritmo do vizinho mais próximos (KNN, presente em 10\% dos artigos lidos), Algoritmo de Naive Bayes (também presente em 10\%) e sistemas Neuro Fuzzy (encontrados em 15\% dos estudos). O KNN realiza a classificação de uma amostra baseada nas amostras vizinhas de um conjunto de treinamento, enquanto o Naive Bayes é um classificador probabilístico baseado no Teorema de Bayes e por fim, os sistemas Neuro Fuzzy fazem a combinação de elementos da lógica difusa (lógica fuzzy) e redes neurais para gerar associações entre as variáveis do problema. [Pintelas et al. 2018].

Na Figura 2 é possível observar as demais técnicas e algoritmos. Para o transtorno de ansiedade, as técnicas mais utilizadas podem ser vistas na Figura 3, enquanto na Figura 4 são apresentadas as técnicas em estudos sobre depressão e por fim, na Figura 5 é possível vizualizar as mais utilizadas em estudos com foco em estresse.

\subsection{Quais os instrumentos mais utilizados para identificar transtornos mentais?}

No que diz respeito aos instrumentos, pode-se perceber que para ansiedade o Inventário Traço-Estado (IDATE) [Spielberger 2010] foi o mais utilizado. Além do IDATE, 


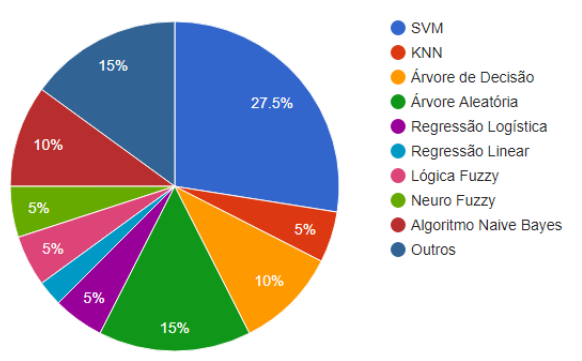

Figura 2. Porcentagem Geral de Algoritmos e Técnicas utilizados

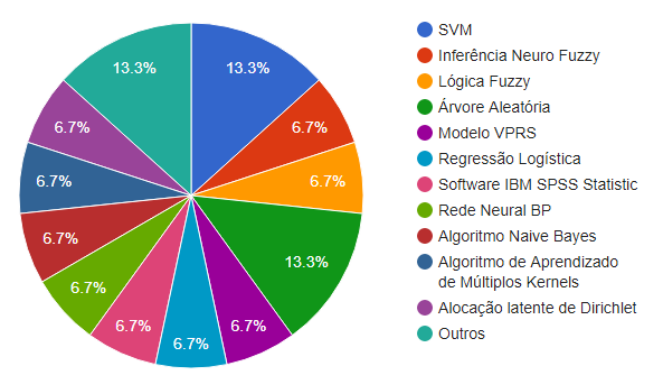

Figura 3. Porcentagem de Algoritmos e Técnicas utilizados em transtornos de Ansiedade

também foram utilizados a Escala de Avaliação de Sintomas (SCL-90) [Derogatis 1992], Teste Abrangente de ansiedade de Sinha (SCAT) [Sinha 1995], Inventário de Personalidade de Maudsley (MPI) [Jensen 1958], Escala de Ansiedade e Estresse (DASS21) [Lovibond and Lovibond 1996] e Questionário de Saúde do Paciente (PHQ-9) [Spitzer et al. 1999]. Para o estresse não houve instrumento predominante, porém os utilizados foram a Escala de Estresse Percebido (PSS-14) [Cohen et al. 1983], Escala de Ansiedade, Depressão e Estresse 21 (DASS-21) [Lovibond and Lovibond 1996], PSS-14 [Cohen et al. 1983].

Em relação a depressão, os instrumentos mais recorrentes foram o Questionário de Saúde do Paciente (PHQ-9) [Spitzer et al. 1999] e o Inventário de Depressão de Beck (BDI-II) [Beck et al. 1996]. A Escala de Avaliação de Sintomas (SCL-90) [Derogatis 1992], DASS-21 [Lovibond and Lovibond 1996] também foram identificados.

\subsection{Quais as variáveis associadas a cada transtorno mental?}

Para resolver essa questão de pesquisa, foi feita uma análise dos artigos com os transtornos mais recorrentes: ansiedade e depressão. Assim, para o transtorno de ansiedade, as variáveis associadas encontradas foram: neuroticismo e extroversão [Devi et al. 2016], enquanto em [Bian et al. 2017] e [Zivin et al. 2009] traz variáveis demográficas como sexo, raça/etnia, idade, e tipo de aluno (graduação ou pós-graduação).[Day et al. 2013], traz as mesmas variáveis associadas, com exceção de tipo de aluno.

Das variáveis relacionadas à depressão, pode-se citar hábitos de leitura e informações demográficas [Hou et al. 2016]. Além disso, outras variáveis associadas a esse transtorno são: sono, cansaço, apetite, concentração, desinteresse ou desânimo para realizar atividades, abatimento, deprimido ou sem esperança, sentir-se mal consigo 


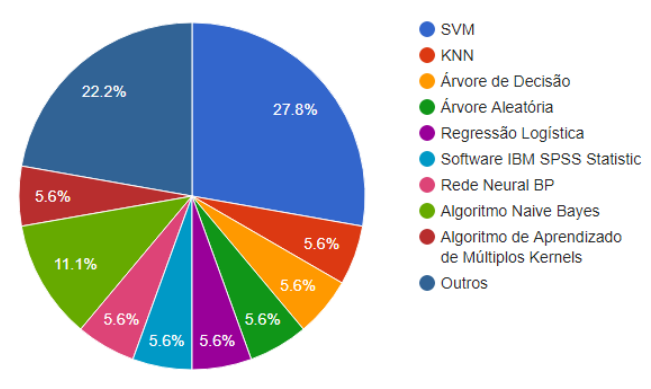

Figura 4. Porcentagem de Algoritmos e Técnicas utilizados em estudos com depressão

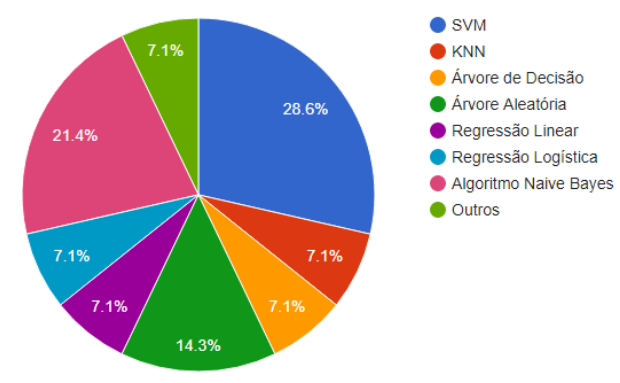

Figura 5. Porcentagem de Algoritmos e Técnicas utilizados em estudos com alteração nos níveis de estresse

mesmo [Miranda and Scoppetta 2018], relações sociais, irritabilidade e raiva, síndrome pré-menstrual (TPM) e humor [Day et al. 2013].

Por fim, para estresse, as variáveis são: Tempo com olhos abertos e fechado [Khosrowabadi et al. 2011], interferência no tempo de reação de uma tarefa, comer, cantar, trabalho de casa, conversas sociais [Egilmez et al. 2017], tempo gasto na internet e pressão no exame [Ahujaa and Bangab 2019].

\subsection{Qual a nacionalidade dos participantes das amostras?}

A figura 6 mostra que as amostras são predominantemente da China e Estados Unidos. A taxa de incidência de transtornos mentais nesses países pode ser uma explicação para esse resultado, pois em 2017 3,31\% da população chinesa apresentava depressão, enquanto 3,03\% ansiedade e nos EUA 4,84\% apresentava depressão e 6,64\% ansiedade [Ritchie and Roser 2019]. Além disso, pode-se observar que apesar de e 6,07\% da população brasileira apresentar algum nível de ansiedade e 3,3\% sofrer com distúrbios relacionados à depressão[WHO et al. 2017], existe escassez de estudos sobre a aplicação de técnicas de mineração de dados para investigação da saúde mental em estudantes universitários.

\section{Conclusões}

O presente estudo teve o objetivo de fazer uma Revisão Sistemática da literatura acerca da utilização de técnicas de mineração de dados para investigação de transtornos mentais em estudantes universitários. As bases de dados eletrônicas utilizadas foram Science Direct e 


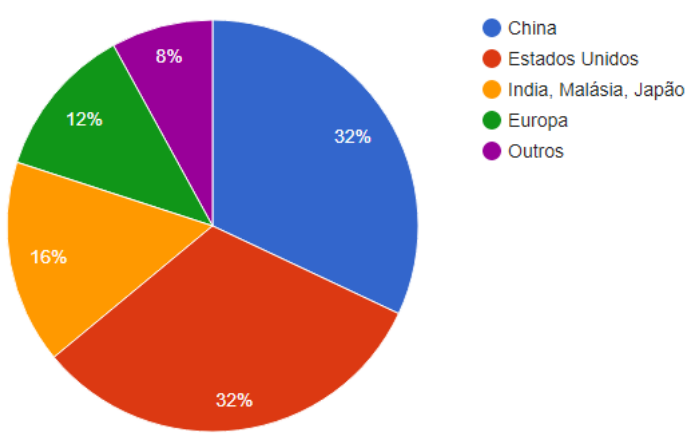

Figura 6. Nacionalidade das amostras

o IEEE. O procedimento de seleção de estudos foi dividido em três etapas, sendo que ao final obteve-se um total de 25 artigos analisados.

Através dos resultados obtidos, pode-se observar escassez de estudos sobre a aplicação de técnicas de mineração de dados em pesquisas de saúde mental em estudantes brasileiros. Além disso, foi possível identificar a nacionalidade das amostras, os instrumentos utilizados e a preferência pela utilização de algoritmos de aprendizagem supervisionada, sendo os Algoritmos SVM e Florestas Aleatórias os mais utilizados. Em relação aos transtornos mentais, nota-se a predominância de depressão, ansiedade e estresse, este último apesar de não se caracterizar como um transtorno propriamente dito, é um fenômeno de interesse dos pesquisadores da área.

Os resultados obtidos apresentam um panorama do estado da arte das aplicações de técnicas de mineração de dados para anlisar a saúde de estudantes universitários. Para trabalhos futuros, uma análise comparativa entre os algoritmos de mineração de dados com base na acurácia pode ser considerada. Outra alternativa seria aplicar técnicas de mineração de dados com o intuito de investigar a saúde mental de estudantes universitários brasileiros, tendo em vista a escassez de trabalhos encontrados com amostras brasileiras.

\section{Referências}

Ahujaa, R. and Bangab, A. (2019). Mental stress detection in university students using machine learning algorithms. Procedia Computer Science, 152:349-353.

Alonso, S. G., de La Torre-Díez, I., Hamrioui, S., López-Coronado, M., Barreno, D. C., Nozaleda, L. M., and Franco, M. (2018). Data mining algorithms and techniques in mental health: a systematic review. Journal of medical systems, 42(9):161.

Association, A. P. et al. (2014). DSM-5: Manual diagnóstico e estatístico de transtornos mentais. Artmed Editora.

Beck, A., Steer, R., and Brown, G. (1996). Manual for the beck depression inventory second edition (bdi-ii) psychological corporation. San Antonio, TX.

Bian, J., Barnes, L. E., Chen, G., and Xiong, H. (2017). Early detection of diseases using electronic health records data and covariance-regularized linear discriminant analysis. In 2017 IEEE EMBS International Conference on Biomedical \& Health Informatics (BHI), pages 457-460. IEEE.

BRASIL, O. (2016). Depressão: o que você precisa saber. 
Cohen, S., Kamarck, T., and Mermelstein, R. (1983). A global measure of perceived stress. Journal of health and social behavior, pages 385-396.

Cui Yuan (2014). Data mining techniques with its application to the dataset of mental health of college students. In 2014 IEEE Workshop on Advanced Research and Technology in Industry Applications (WARTIA), pages 391-393.

da Victoria, M. S., Bravo, A., Felix, A. K., Neves, B. G., Rodrigues, C. B., Ribeiro, C. C. P., Canejo, D., Coelho, D., Sampaio, D., Esteves, I. M., et al. (2015). Níveis de ansiedade e depressão em graduandos da universidade do estado do rio de janeiro (uerj). Encontro: Revista de Psicologia, 16(25):163-175.

Day, V., McGrath, P. J., and Wojtowicz, M. (2013). Internet-based guided self-help for university students with anxiety, depression and stress: a randomized controlled clinical trial. Behaviour research and therapy, 51(7):344-351.

de Rezende, C., Abrão, C., Coelho, E., and da Silva Passos, L. (2008). Prevalência de sintomas depressivos entre estudantes de medicina da universidade federal de uberlândia. Rev Bras Educ Med, 32(3):315-23.

Derogatis, L. (1992). Administration, scoring and procedures manual-ii (baltimore, md: Clinical psychometric research)(scl-90-r).

Devi, S., Kumar, S., and Kushwaha, G. S. (2016). An adaptive neuro fuzzy inference system for prediction of anxiety of students. In 2016 Eighth International Conference on Advanced Computational Intelligence (ICACI), pages 7-13. IEEE.

Egilmez, B., Poyraz, E., Zhou, W., Memik, G., Dinda, P., and Alshurafa, N. (2017). Ustress: Understanding college student subjective stress using wrist-based passive sensing. In 2017 IEEE International Conference on Pervasive Computing and Communications Workshops (PerCom Workshops), pages 673-678. IEEE.

Han, J. and Kamber, M. (2005). Data mining : concepts and techniques. Kaufmann, San Francisco [u.a.].

Hou, Y., Xu, J., Huang, Y., and Ma, X. (2016). A big data application to predict depression in the university based on the reading habits. In $20163 \mathrm{rd}$ International Conference on Systems and Informatics (ICSAI), pages 1085-1089. IEEE.

Jensen, A. R. (1958). The maudsley personality inventory. Acta Psychologica, 14:314325.

Khosrowabadi, R., Quek, C., Ang, K. K., Tung, S. W., and Heijnen, M. (2011). A braincomputer interface for classifying eeg correlates of chronic mental stress. In The 2011 International Joint Conference on Neural Networks, pages 757-762. IEEE.

Lovibond, S. H. and Lovibond, P. F. (1996). Manual for the depression anxiety stress scales. Psychology Foundation of Australia.

Miranda, C. A. C. and Scoppetta, O. (2018). Factorial structure of the patient health questionnaire-9 as a depression screening instrument for university students in cartagena, colombia. Psychiatry research, 269:425-429.

OMS, O. M. d. S. (2002). Relatório mundial de saúde: Saúde mental, nova concepção, nova esperança. 
Pintelas, E. G., Kotsilieris, T., Livieris, I. E., and Pintelas, P. (2018). A review of machine learning prediction methods for anxiety disorders. In Proceedings of the 8th International Conference on Software Development and Technologies for Enhancing Accessibility and Fighting Info-exclusion, pages 8-15.

Prince, M., Patel, V., Saxena, S., Maj, M., Maselko, J., Phillips, M. R., and Rahman, A. (2007). No health without mental health. The lancet, 370(9590):859-877.

Ritchie, H. and Roser, M. (2019). Mental health. Our World in Data. https://ourworldindata.org/mental-health.

Sinha, A. (1995). Manual for sinha's comprehensive anxiety test (scat). Agra: National Psychological Corporation.

Spielberger, C. D. (2010). State-trait anxiety inventory. The Corsini encyclopedia of psychology, pages 1-1.

Spitzer, R. L., Kroenke, K., Williams, J. B., Group, P. H. Q. P. C. S., et al. (1999). Validation and utility of a self-report version of prime-md: the phq primary care study. Jama, 282(18):1737-1744.

Srividya, M., Mohanavalli, S., and Bhalaji, N. (2018). Behavioral modeling for mental health using machine learning algorithms. Journal of medical systems, 42(5):88.

WHO et al. (2017). Depression and other common mental disorders: global health estimates. Technical report, World Health Organization.

Yuan, C. (2014). Data mining techniques with its application to the dataset of mental health of college students. In 2014 IEEE Workshop on Advanced Research and Technology in Industry Applications (WARTIA), pages 391-393. IEEE.

Zivin, K., Eisenberg, D., Gollust, S. E., and Golberstein, E. (2009). Persistence of mental health problems and needs in a college student population. Journal of affective disorders, 117(3):180-185. 